\title{
Full protection of superconducting qubit systems from coupling errors
}

\author{
M. J. Storcz, ${ }^{1, *}$ J. Vala, ${ }^{2}$ K. R. Brown, ${ }^{2}$ J. Kempe,${ }^{2, \dagger}$ F. K. Wilhelm, ${ }^{1}$ and K. B. Whaley ${ }^{2}$ \\ ${ }^{1}$ Physics Department, ASC, and CeNS, Ludwig-Maximilians-Universität, Theresienstr. 37, 80333 München, Germany \\ ${ }^{2}$ Department of Chemistry and Pitzer Center for Theoretical Chemistry, University of California, Berkeley, California 94720, USA
}

(Received 18 April 2005; published 12 August 2005)

\begin{abstract}
Solid state qubits realized in superconducting circuits are potentially scalable. However, strong decoherence may be transferred to the qubits by various elements of the circuits that couple individual qubits, particularly when coupling is implemented over long distances. We propose here an encoding that provides full protection against errors originating from these coupling elements, for a chain of superconducting qubits with a nearest neighbor anisotropic $X Y$-interaction. The encoding is also seen to provide partial protection against errors deriving from general electronic noise.
\end{abstract}

DOI: $10.1103 /$ PhysRevB.72.064511

PACS number(s): 03.67.Pp, 03.65.Yz, 03.67.Lx, 85.25.-j

Superconducting flux qubits have been shown to possess many of the necessary features of a quantum bit (qubit), including the ability to prepare superpositions of quantum states $^{1,2}$ and manipulate them coherently. ${ }^{3}$ In these systems, the dominating error source appears to be decoherence due to flux noise. ${ }^{4}$ Present designs for arrays of multiple flux qubits that are coupled through their flux degree of freedom are easily implemented from an experimental point of view. ${ }^{5}$ However, when scaling up to large numbers of qubits, they suffer from technical restrictions such as possible flux crosstalk and a need for physically large coupling elements, which are expected to act as severe antennas for decoherence. The possibility of avoiding errors by prior encoding into decoherence free subspaces (DFS) that are defined by the physical symmetries of the qubit interaction with the environment is consequently very attractive. Such encoding is also attractive for superconducting charge qubits, ${ }^{6,7}$ which are subject to similar decoherence sources. ${ }^{8}$

In this work, we show how to develop such protection for qubits coupled by the nearest neighbor $X Y$-interaction that is encountered in both flux and charge qubit designs. ${ }^{9}{ }^{910} \mathrm{We}$ demonstrate that for this coupling, a two-qubit encoding into a DFS provides full protection against noise from the coupling elements. Moreover, all encoded single-qubit operations are also protected from collective decoherence deriving from the electromagnetic environment. The protection is seen to result from a combination of symmetry in the coupling element and a restricted environmental phase space of the multi-qubit system - the DFS alone would not be sufficient. The analysis makes use of an exact unitary transformation of $1 / f$ phase noise in the coupling element (hence with a sub-Ohmic power spectrum) into regular nearestneighbor correlated flux noise on the qubits that is characterized by a super-Ohmic power spectrum. To assess the performance of the encoding we add to this coupling-derived noise a single-qubit Ohmic noise source that represents the generic uncorrelated environmental factors and analyze the fidelity of encoded quantum gate operations.

The Hamiltonian of a linear chain of $X Y$ coupled qubits reads

$$
\begin{aligned}
\mathbf{H}_{q} & =\mathbf{H}_{0}+\mathbf{H}_{\mathrm{int}} \\
& =\sum_{i}\left[\epsilon_{i} \hat{\sigma}_{z}^{(i)}+\Delta_{i} \hat{\sigma}_{x}^{(i)}+K_{i, i+1}\left(\hat{\sigma}_{x}^{(i)} \hat{\boldsymbol{\sigma}}_{x}^{(i+1)}+\hat{\sigma}_{y}^{(i)} \hat{\sigma}_{y}^{(i+1)}\right)\right],
\end{aligned}
$$

where $\mathbf{H}_{0}=\Sigma_{i}\left[\epsilon_{i} \hat{\sigma}_{z}^{(i)}+\Delta_{i} \hat{\sigma}_{x}^{(i)}\right]$ is the uncoupled qubit Hamiltonian, and $K_{i, i+1}$ is the strength of the inter-qubit coupling, $\mathbf{H}_{\text {int }}$. We assume that it is possible to switch the coupling $K_{i, i+1}$ and the flux bias $\epsilon_{i}\left(\Phi_{x, i}\right)$ of each qubit separately. Such a Hamiltonian can be realized using flux qubits with capacitive coupling. ${ }^{10}$ The switch for this interaction can in principle be implemented using PIN varactor diodes, micromechanical devices, or small Josephson junctions. ${ }^{11}$ Switching on the coupling suppresses the tunnel amplitudes ${ }^{10} \Delta_{i}$. The Hamiltonian of Eq. (1) can also be readily implemented in charge qubits, i.e., Cooper pair boxes coupled by Josephson junctions, ${ }^{9}$ whose coupling strength can be tuned through an external magnetic field. In both cases, the couplers are large objects and hence act as efficient antennas for charge and/or flux noise when the coupling is on. When the coupling is switched off, this noise is confined within the coupler and does not affect the qubits.

The decoherence sources relevant to Eq. (1) are background charges. This can be represented as $1 / f$ noise in the coupler as we explain below. In addition general electromagnetic (e.m.) noise, both local flux or electronics noise, couples to single qubits and, for long wavelength, also to multiple qubits. The e.m. noise is represented as usual by Ohmic noise which has both uncorrelated and collective components. The effect of these environmental decoherence sources on Eq. (1) is represented by the usual (linear) coupling to a bath of oscillators $\mathbf{H}_{b}=\sum_{i}\left(a_{i}^{\dagger} a_{i}+\frac{1}{2}\right)$, characterized by a spectral density $J(\omega)=\Sigma_{i}\left|\lambda_{i}\right|^{2} \delta\left(\omega-\omega_{i}\right)$, with the coupling strength characterized by a dimensionless parameter ${ }^{12}$ $\alpha$.

We first show how the coupling and local noise are described in this framework. Background charge fluctuations $\delta q(t)$ arising in the capacitive coupling elements between qubits $i$ and $i+1$, induce geometric Aharonov-Casher ${ }^{13}$ phases $\delta \phi(t) \propto \delta q(t)$ when the qubit flux states tunnel between eigenstates of $\hat{\sigma}_{z}$. This results in a correlated two-qubit 
error operator $\exp \left[i \delta \phi\left(\hat{\sigma}_{z}^{(i)}+\hat{\sigma}_{z}^{(i+1)}\right)\right]$ acting on $\mathbf{H}_{\mathrm{q}}$. The lowfrequency limit of this phase noise in the coupling elements can be approximated as a Gaussian $1 / f$ noise process deriving from coupling to a sub-Ohmic oscillator bath with associated spectral density ${ }^{12,13} \quad J_{\phi}^{2 q b}(\omega)=\left(\alpha_{0} / \epsilon_{0}\right) \operatorname{sign}(\omega) e^{-\omega / \omega_{c}}$. Here and henceforth we set $\hbar, k_{B}=1$. This leads to a classical power spectrum in the frequency domain

$$
\begin{aligned}
S_{\phi}(\omega) & =\frac{1}{2}\langle\delta \phi(t) \delta \phi(0)+\delta \phi(0) \delta \phi(t)\rangle_{\omega} \\
& =J_{\phi}^{2 q b}(\omega) \operatorname{coth}(\omega / 2 T) \\
& \simeq\left(2 T \alpha_{0} / \omega_{c} \omega\right)
\end{aligned}
$$

for $\omega \ll T$, which characterizes the environmental phase space of the correlated two-qubit errors due to capacitive coupling. Uncorrelated single qubit errors deriving from local electronic elements are represented here by bath coupling to the flux states, i.e., $\hat{\sigma}_{z}$ errors. This is typically represented by a bath having an Ohmic spectral density, ${ }^{14} J_{\epsilon, \Omega}^{1 q b}$ $=\alpha_{\Omega} \omega \omega_{c}^{2} /\left(\omega_{c}^{2}+\omega^{2}\right)$, which thus characterizes the environmental phase space of the uncorrelated single-qubit errors. We note that very recently, $\hat{\sigma}_{x}$ single-qubit errors (i.e., bit flip errors) have also been identified..$^{15}$ The third source of errors, correlated errors deriving from long wavelength electromagnetic radiation, can be removed by encoding into a DFS as we show below, independent of the form of the spectral density associated with the source of such collective decoherence.

We can formally introduce the noise due to background charges into the total Hamiltonian $\mathbf{H}_{q}+\mathbf{H}_{b}$ by transforming the total Hamiltonian with a unitary operator $U_{q b}$ $=\exp \left[i \delta \phi\left(\hat{\sigma}_{z}^{(i)}+\hat{\sigma}_{z}^{(i+1)}\right)\right]$, resulting in

$$
\mathbf{H}=\mathbf{H}^{\prime}+\mathbf{H}_{b}=U_{q b} \mathbf{H}_{q} U_{q b}^{\dagger}+\mathbf{H}_{b},
$$

with associated spectral density $J_{\phi}^{2 q b}(\omega)$. Thus, the error acts in the interaction picture as a time-dependent unitary transformation and it can be eliminated by undoing the transformation. In NMR (nuclear magnetic resonance) language, this is a transformation to the "co-fluctuating" frame. The unitary transformation is properly undone by a time-dependent unitary transformation in the interaction picture, which transforms the states as $\left|\psi^{\prime}\right\rangle=U_{q b}^{\dagger}|\psi\rangle$ and the coupled Hamiltonian as

$$
\begin{gathered}
\mathbf{H}_{\mathrm{eff}}=U_{q b}^{\dagger} \mathbf{H} U_{q b}-i U_{q b}^{\dagger} \frac{d}{d t} U_{q b}, \\
-i U_{q b}^{\dagger} \frac{d}{d t} U_{q b}=\frac{1}{2}\left[\hat{\sigma}_{z}^{(i)}+\hat{\sigma}_{z}^{(i+1)}\right] \delta \dot{\phi} .
\end{gathered}
$$

The last term is understood as an effective system-bath interaction, written more explicitly

$$
\mathbf{H}_{\mathrm{SB}}=-i U_{q b}^{\dagger} \frac{d}{d t} U_{q b}=\frac{1}{2}\left[\hat{\sigma}_{z}^{(i)}+\hat{\sigma}_{z}^{(i+1)}\right] \otimes \sum_{n} i \omega_{n} \lambda_{n}\left(a_{n}-a_{n}^{\dagger}\right) .
$$

Note that $\mathbf{H}_{\mathrm{q}}=U_{q b}^{\dagger} \mathbf{H}^{\prime} U_{q b}$. Physically, this arises from the transformation into the noninertial co-fluctuating frame as an inertial force. It is recognized that (6) is the regular spin boson coupling $\mathbf{H}_{\mathrm{SB}, \text { eff }}=\sum_{i}\left(\lambda_{i}^{\prime} a_{i}+\lambda_{i}^{\prime *} a_{i}^{\dagger}\right)$ with $\lambda_{i}^{\prime}=i \omega \lambda_{i}$. In this transformed representation we now have correlated flux errors, i.e., pairwise coupling of the qubit $\hat{\sigma}_{z}$ operators to energy fluctuations given by the time-derivative of the fluctuating correlated coupler phase, $\delta \dot{\phi}$. Most importantly, the associated spectral density of the oscillator bath is also transformed, becoming $J_{\epsilon}^{2 q b}(\omega)=\omega^{2} J_{\phi}^{2 q b}(\omega)=\alpha_{0} \omega^{2} \operatorname{sign}(\omega) / \epsilon_{0}$, which is now super-Ohmic. Similar arguments can be applied to the flux noise arising when two charge qubits are coupled by a SQUID, except that here the coupling (flux) noise is usually Ohmic rather than sub-Ohmic, so that the transformed spectral density is proportional to $\omega^{3}$ rather than to $\omega^{2}$. Note, that the flux states only get transformed by phase factors, hence computation and measurement carried out in this basis are unaffected by this transformation.

To protect against these correlated errors we employ a two-qubit encoding $|0\rangle_{L}=|01\rangle,|1\rangle_{L}=|10\rangle$ which is recognizable as the smallest DFS encoding that can protect against collective dephasing. ${ }^{16}$ It, therefore, automatically protects against any correlated phase errors, including our third source of error deriving from long wavelength e.m. noise.

We will show that as a result of the symmetry in the bath, in particular, because of the form of its spectral density, this encoding also provides complete protection against the noise arising during capacitive coupling. This results in perfect performance of both encoded single qubit and two qubit operations when correlated errors during two-qubit operations are the only source of decoherence. Uncorrelated single qubit errors are then the only remaining mechanism leading to a reduced fidelity of quantum gates. We find below that for single qubit errors of less than or equal strength to two qubit errors, the DFS encoding still provides a significant, although now incomplete, protection.

The two logical qubits are encoded into four physical qubits using the encoding scheme $|00\rangle_{L}=|0101\rangle_{P},|01\rangle_{L}$ $=|0110\rangle_{P},|10\rangle_{L}=|1001\rangle_{P},|11\rangle_{L}=|1010\rangle_{P}$, where $L$ and $P$ denote logical and physical states, respectively. We assume that the four physical qubits constitute a linear array (this need not be contiguous) which we label 1,2,3,4. This fourdimensional subspace is left invariant by collective errors involving qubits 1 and $2, U_{q b}$ with $i=1$, as well as by errors involving qubits 3 and $4, U_{q b}$ with $i=3$, but not by collective errors involving qubits 2 and 3, i.e., $U_{q b}$ with $i=2$, see Ref. 16. A simple counting argument shows that a DFS that protects against all two-qubit errors including those between the two encoded qubits does not exist.

The latter errors arise when switching on the coupling between qubits 2 and 3 with $\mathbf{H}_{\text {int }}$ as described above, in order to perform logical two- qubit operations. Thus, in a Hamiltonian formulation within the basis spanned by the encoded subspace vectors, the coupling error $\hat{\sigma}_{z}^{(2)}+\hat{\sigma}_{z}^{(3)}$ between qubits 2 and 3 does not have identical degenerate eigenvalues as would be required for a DFS. ${ }^{17}$ Moreover, the physical single qubit errors also do not fulfill this requirement of degenerate eigenvalues. In the language of quantum error correcting codes $(\mathrm{QECC})^{18}$ where a DFS is a particular example of a degenerate $\mathrm{QECC}^{16}$ we, therefore, conclude that our encoding is not fully degenerate under the action of both the correlated two-qubit and single qubit errors and does not 
constitute a true DFS for both classes of errors. A true DFS would be completely degenerate, giving identical syndromes of unity for all of these errors. As a result of this lack of degeneracy, additional operations are in principle needed for correction of the uncorrelated noise, i.e., of the physical single-qubit errors, as well as for correction of the coupling error $U_{q b}, i=2$. Nevertheless, we will see below that the latter coupling errors resulting from the background charge fluctuations are actually suppressed by the bath properties and its symmetry after application of the transformation $U_{q b}$, so that only the single qubit errors need to be actively corrected.

The encoded single-qubit operations, given here without loss of generality for the first encoded logical qubit only, can be shown to be

$$
\begin{gathered}
e^{-i \bar{\sigma}_{z}^{(1)} \tau}=e^{-i \hat{\sigma}_{z}^{(2)} \tau} \\
e^{-i \bar{\sigma}_{x}^{(1)} \tau}=e^{-i \tilde{\mathbf{H}}_{\mathrm{int}}^{12} \tau} \\
e^{-i \bar{\sigma}_{y}^{(1)} \tau}=e^{i \bar{\sigma}_{z}^{(1)} \frac{\pi}{4}} e^{i \bar{\sigma}_{x}^{(1)} \tau} e^{-i \bar{\sigma}_{z}^{(1)} \frac{\pi}{4}},
\end{gathered}
$$

where $\tilde{\mathbf{H}}_{\text {int }}=\left(\mathbf{H}_{\text {int }} / \epsilon_{0}\right)$ and $\tau=t \epsilon_{0}$. The first operation is straightforwardly achieved by tuning the flux bias. To implement the second operation, $\bar{\sigma}_{x}^{(1)}$, we need to cancel the effect of $\mathbf{H}_{0}$. This is also straightforward, if $\epsilon_{i}$ and $\Delta_{i}$ can be tuned to zero. If $\Delta$ cannot be tuned to zero, it is nevertheless still possible to act with $\mathbf{H}_{\text {int }}$ alone, by combining a short time Trotter expansion with operator conjugation as follows. First, we recognize that conjugation of $\mathbf{H}_{q}$ with $\hat{\sigma}_{z}$ can invert the sign of $\Delta_{i}$

$$
e^{-i \mathbf{H}_{q}\left(-\Delta_{1},-\Delta_{2}\right) t}=e^{-i\left(\hat{\sigma}_{z}^{(1)}+\hat{\sigma}_{z}^{(2)}\right) \pi / 2} e^{-i \mathbf{H}_{q}\left(\Delta_{1}, \Delta_{2}\right) t} e^{i\left(\hat{\sigma}_{z}^{(1)}+\hat{\sigma}_{z}^{(2)}\right) \pi / 2} .
$$

The alternation of $\mathbf{H}_{q}\left(\Delta_{1}, \Delta_{2}, K_{12}\right)$ with $\mathbf{H}_{q}\left(-\Delta_{1},-\Delta_{2}, K_{12}\right)$ results in the desired action of $\mathbf{H}_{\text {int }}$, up to commutator errors between $\mathbf{H}_{\text {int }}$ and $\Delta \hat{\sigma}_{x}^{(i),(j)}$ which can be suppressed by making a Trotter expansion:

$$
\lim _{n \rightarrow \infty}\left(e^{-i \mathbf{H}_{q}\left(\Delta_{1}, \Delta_{2}, K_{12}\right) t / 2 n} e^{-i \mathbf{H}_{q}\left(-\Delta_{1},-\Delta_{2}, K_{12}\right) t / 2 n}\right)^{n}=e^{-i \tilde{\mathbf{H}}_{\mathrm{int}}^{12} \tau} .
$$

This scheme requires only relatively small values of $n$ to be effective. Direct simulation shows that for $n \sim 10$, the relative deviation of individual matrix elements $U_{n m}$ from $U_{n m}^{\text {ideal }}$ is smaller than $1 \%$. During all these encoded single qubit operations the encoded qubit remains in the DFS encoded subspace and so is fully protected against correlated twoqubit errors deriving from both the capacitive coupling and from any other electromagnetic correlated noise.

Encoded two-qubit operations require pairwise coupling of physical qubits from the two encoded qubits $|0\rangle_{L}$ and $|1\rangle_{L}$, e.g., qubits 2 and 3 as mentioned above. The encoded $\bar{U}_{z z}(t)$ two-qubit controlled-phase operation is

$$
\bar{U}_{z z}(t)=e^{-i \bar{\sigma}_{z}^{(1)} \bar{\sigma}_{z}^{(2)}}=e^{i S_{x} \frac{\pi}{4}} e^{i \tilde{\mathbf{H}}_{\mathrm{int}}^{23} \tau / 2} e^{-i \sigma_{x}^{(2)} \frac{\pi}{2}} e^{i \tilde{\mathbf{H}}_{\mathrm{int}}^{23} / 2} e^{i S_{x}^{\prime} \frac{\pi}{4}},
$$

where $S_{x}^{\prime}=\hat{\sigma}_{x}^{(2)}-\hat{\sigma}_{x}^{(3)}$ and $S_{x}=\hat{\sigma}_{x}^{(2)}+\hat{\sigma}_{x}^{(3)}$. This can be combined with an encoded single qubit Hadamard gate to produce the controlled NOT (CNOT) gate. ${ }^{14}$ Now the first element of $\bar{U}_{z z}(t), e^{i S_{x}^{\prime} \pi / 4}$, takes the DFS states outside the subspace to form superpositions of DFS and non-DFS states and populate the non-DFS states $|0111\rangle,|0100\rangle,|1011\rangle$ and $|1000\rangle$. Detailed analysis reveals that the two-qubit operation Eq. (12) will always take the encoded qubits out of the DFS encoded subspace. However, during these excursions out of the DFS, when only coupling errors are present, only pure dephasing processes which do not flip eigenstates can contribute to decoherence, ${ }^{14}$ since the coupling to the bath commutes with the interqubit coupling. The rates of these dephasing processes are proportional to $S(0)$ $=\lim _{\omega \rightarrow 0} J_{\epsilon}^{2 q b}(\omega) \operatorname{coth}(\omega / 2 T)$, which vanishes as a result of the super-Ohmic shape of $J_{\epsilon}^{2 q b}$ derived from the tunnelingflux transformation introduced above. Consequently these processes "lack phase space" in the environmental degrees of freedom and hence are fully suppressed. This excursion out of the DFS encoded subspace into a larger region of the full Hilbert space in which only pure dephasing processes contribute to the decoherence can alternatively be viewed as an excursion into a larger subspace that is characterized by suppression of relaxation processes.

We demonstrate the benefits of the DFS encoding by numerical studies of the CNOT gate, calculated from the simulated evolution of the reduced density matrix for the coupled flux qubits using the Bloch-Redfield description of the spinboson model of the qubit and its bath coupling characterized by ${ }^{14} J(\omega)$. This approach is valid for $\alpha_{0}, \alpha_{\Omega} \ll 1$. To quantify the gate performance we evaluate the fidelity ${ }^{19} \mathcal{F}$ of the encoded quantum gate operation, defined by $\mathcal{F}$ $=\frac{1}{16} \sum_{j=1}^{16}\left\langle\Psi_{i n}^{j}\left|U_{G}^{+} \rho_{G}^{j} U_{G}\right| \Psi_{i n}^{j}\right\rangle$. Here $U_{G}$ is the unitary matrix describing the desired ideal gate, and $\rho_{G}^{j}=\rho\left(t_{G}\right)$ is the density matrix obtained from attempting a quantum gate operation in a hostile environment, i.e., with errors, evaluated for all initially unentangled product states ${ }^{19}$ from the encoded logical basis, $\rho(0)=\left|\Psi_{i n}^{j}\right\rangle\left\langle\Psi_{i n}^{j}\right|$. The states $\left|\Psi_{i n}^{j}\right\rangle$ are defined in Ref. 20.

Figure 1 shows the calculated gate fidelity for an encoded CNOT operation $\bar{U}_{\text {CNOT }}$, obtained from $\bar{U}_{z z}$ together with the relevant encoded single qubit gates. We see that, as predicted by the above analysis, when only two-qubit errors are active $\left(\alpha^{1 \mathrm{qb}}=0\right)$ the gate performance is perfect. When additional uncorrelated single-qubit errors during single qubit operations occur $\left(\alpha^{1 \mathrm{qb}}\right)$, the gate fidelity is seen to decrease as the strength of these errors increases. The DFS encoding is thus seen to give $100 \%$ protection against the primary coupling errors in addition to correlated background errors. It does not protect against uncorrelated single qubit errors, in fact, due to the larger overhead, DFS encoding alone is sensitive against these (compare sets I and II). However, the uncorrelated single qubit errors can be well treated by active quantum error correction, particularly if the error rates for single 


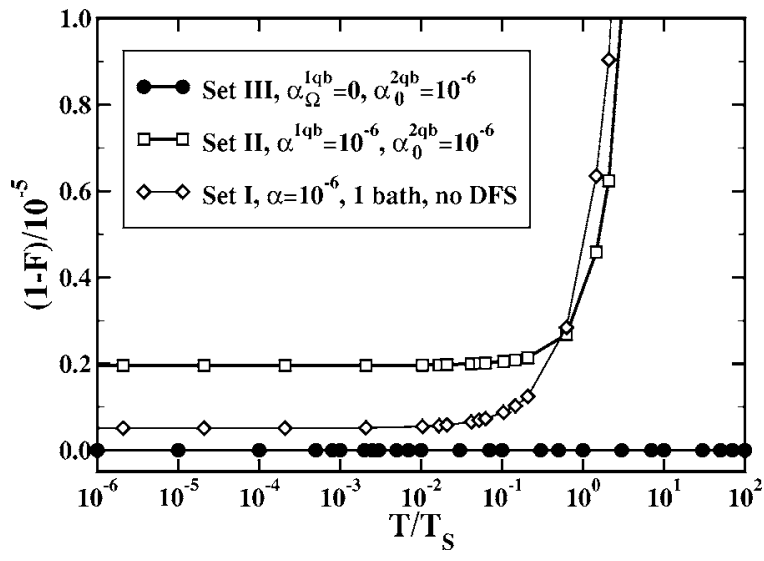

FIG. 1. Fidelity deviation $1-\mathcal{F}$ vs temperature for the encoded CNOT operation, shown for two different combinations of superOhmic two-qubit noise (strength $\alpha^{2 q \mathrm{~b}}=\alpha_{0}$ ) and Ohmic single-qubit noise (strength $\alpha^{1 q \mathrm{~b}}=\alpha_{\Omega}$ ) acting on the physical qubits. The characteristic temperature scale is $T_{s}=\epsilon_{0}\left(h / k_{B}\right)$, yielding $T_{s}=48 \mathrm{mK}$ for qubits with energies $\epsilon_{i}=\epsilon_{0} \equiv 1 \mathrm{GHz}, i=1,2$. Here, $\epsilon_{0}$ is used as an energy unit for the correlation function. Solid lines are provided as guides to the eye. Ideal gate performance is achieved when $\alpha^{1 q b}$ $=0$. Detailed analysis shows that the fidelity depends linearly on $\alpha^{1 q b}$. For comparison, set I shows the corresponding performance of the unencoded CNOT operation taken from Ref. 14.

qubit and correlated errors are comparable. It is also possible to combine this encoding scheme with a QECC in order to achieve fault-tolerance. Using the scheme proposed in Ref. 21, the leakage problem of standard QECC methods can be overcome.

Saturation of the gate quality at low temperatures occurs because all decohering processes (except spontaneous emission) are frozen out. This occurs when $k_{B} T \simeq E_{\min }$, where $E_{\text {min }}$ is the lowest energy splitting in the system. Here, $E_{\text {min }}$ $=\epsilon_{0}$. Even during the excursion out of the DFS, transitions between the eigenstates of the Hamiltonian involving spontaneous emission are forbidden by symmetry. Thus, at low temperatures, only energy-conserving "pure dephasing" processes influence the gate. These are proportional to the noise power $S(\omega \rightarrow 0)$. For an Ohmic environment, this noise is purely thermal, ${ }^{14} S(0) \propto T$, so that the gate performance is still limited at any finite temperature. For the super-Ohmic case, $S(0)=0$ at any $T$ (Fig. 1 ). When $\alpha_{\Omega}$ is small, the fidelity can be considerably increased because the errors from the coupling elements introduce no new constraints; i.e., if, for equal coupling strength to the electromagnetic environment, the appropriate relative weight of two qubit errors is larger than that of one qubit errors, it is evident that the DFS encoding provides considerable protection. Thus, for optimizing two-qubit gates it is of crucial importance to identify, whether or not the noise is correlated between qubits. This is a critical challenge for experiment. An experimental signature of correlated noise is, e.g., the superior coherence of the states used as logical qubits in this work.

In conclusion, we have shown that using a DFS encoding of superconducting flux or charge qubits can significantly enhance their gate performance for the entangling two-qubit operations that are required to implement quantum computation. The DFS-encoding proposed here ensures that all encoded single-qubit operations are protected against $1 / f$ noise in the capacitive coupling elements, as well as from correlated electromagnetic noise. The latter are the errors originating from the coupling of the qubits to a common electromagnetic environment. When only the capacitive coupling errors arising during two-qubit operations are present, even though these are not automatically protected by this DFS encoding, we find that perfect fidelity can still be achieved. We have shown that this is a consequence of two symmetries of the bath. First, commutation of the system-bath coupling with the interqubit coupling results in elimination of spontaneous emission between qubit eigenstates. Second, a vanishing bath spectral density for dephasing processes results from the exact correspondence of the $1 / f$ sub-Ohmic charge noise in the coupler to super-Ohmic flux noise on the qubits.

The phase space restriction found here derives from the choice of the $X Y$-interaction between the qubits: coupler noise from other interactions would explore the full phase space during the two-qubit operation. Thus the $X Y$-coupling is a very attractive coupling scheme whenever decoherence is a major concern. From the results presented here, we expect that this DFS-inspired encoding, which is also very efficient, requiring only two physical qubits per logical qubit, will therefore be useful for reducing the noise in quantum circuits based on superconducting qubits.

\section{ACKNOWLEDGMENTS}

We thank T.L. Robertson for useful discussions. This work was supported in part by the NSA and ARDA under ARO contract number P-43385-PH-QC, in part by the National Science Foundation under the ITR program, Grant No. EIA-0205641, and by DFG through SFB 631.

\footnotetext{
*Electronic address: storcz@ theorie.physik.uni-muenchen.de

${ }^{\dagger}$ Also affiliated with CNRS-LRI, UMR 8623, Université de ParisSud, 91405 Orsay, France.

${ }^{1}$ C. H. van der Wal, A. C. J. ter Haar, F. K. Wilhelm, R. N. Schouten, C. J. P. M. Harmans, T. P. Orlando, S. Lloyd, and J. E. Mooij, Science 290, 773 (2000).

${ }^{2}$ J. R. Friedman, V. Patel, W. Chen, S. K. Tolpygo, and J. E. Lukens, Nature (London) 406, 43 (2000).
}

${ }^{3}$ I. Chiorescu, Y. Nakamura, C. J. P. M. Harmans, and J. E. Mooij, Science 299, 1869 (2003).

${ }^{4}$ F. K. Wilhelm, M. J. Storcz, C. H. van der Wal, C. J. P. M. Harmans, and J. E. Mooij, Adv. Solid State Phys. 43, 763 (2003).

${ }^{5}$ J. B. Majer, F. G. Paauw, A. C. J. ter Haar, C. J. P. M. Harmans, J. E. Mooij, Phys. Rev. Lett. 94, 090501 (2005).

${ }^{6}$ Y. A. Pashkin, T. Yamamoto, O. Astafiev, Y. Nakamura, D. V. 
Averin, and J. S. Tsai, Nature (London) 421, 823 (2003).

${ }^{7}$ T. Yamamoto, Yu. A. Pashkin, O. Astafiev, Y. Nakamura, and J. S. Tsai, Nature (London) 425, 941 (2003).

${ }^{8}$ X. Zhou, M. Wulf, Z. Zhou, G. Guo, and M. J. Feldman, Phys. Rev. A 69, 030301(R) (2004); J. Q. You, X. Hu, and F. Nori, preprint arXiv: cond-mat/0407423 (2004).

${ }^{9}$ J. Siewert, R. Fazio, G. M. Palma, and E. Sciacca, J. Low Temp. Phys. 118, 795 (2000).

${ }^{10}$ L. S. Levitov, T. P. Orlando, J. B. Majer, and J. E. Mooij, preprint arXiv: cond-mat/0108266 (2001).

${ }^{11}$ D. V. Averin and C. Bruder, Phys. Rev. Lett. 91, 057003 (2003).

${ }^{12}$ A. Shnirman, Yu. Makhlin, and G. Schön, Phys. Scr., T 102, 147 (2002).

${ }^{13}$ F. K. Wilhelm and J. E. Mooij, unpublished.

${ }^{14}$ M. J. Storcz and F. K. Wilhelm, Phys. Rev. A 67, 042319 (2003).
${ }^{15}$ D. J. Van Harlingen, T. L. Robertson, B. L. T. Plourde, P. A. Reichardt, T. A. Crane, and J. Clarke, Phys. Rev. B 70, 064517 (2004).

${ }^{16}$ J. Kempe, D. Bacon, D. A. Lidar, and K. B. Whaley, Phys. Rev. A 63, 042307 (2001).

${ }^{17}$ D. A. Lidar and K. B. Whaley, in Irreversible Quantum Dynamics, edited by F. Benatti and R. Floreanini (Springer Lecture Notes in Physics, Berlin, 2003), Vol; 622, p.83.

${ }^{18}$ A. M. Steane, in Introduction to Quantum Computation and Information, edited by H. K. Lo, S. Popescu, and T. P. Spiller (World Scientific, Singapore, 1999), p.184.

${ }^{19}$ J. F. Poyatos, J. I. Cirac, and P. Zoller, Phys. Rev. Lett. 78, 390 (1997).

${ }^{20}$ M. Thorwart, P. Hänggi, Phys. Rev. A 65, 012309 (2002).

${ }^{21}$ M. Mohseni, D. A. Lidar, Phys. Rev. Lett. 94, 040507 (2005). 